\title{
Open Branes and Little Strings
}

\author{
Troels Harmark \\ Niels Bohr Institute, Blegdamsvej 17, 2100 Copenhagen, Denmark.
}

\begin{abstract}
This is a short review of the newly discovered ODp-theories that are nongravitational six-dimensional theories defined as the decoupling limit of NS5-branes in the presence of a near-critical $(p+1)$-form RR fields. We discuss the motivation for these new theories, their definitions and properties, and their relation to NCOS theory, OM theory and Little String Theory, focusing on the cases $p=1,2$.
\end{abstract}

\section{Introduction}

This talk is based mainly on the paper [1] and in addition on the papers [2, 3, 4]. The purpose of this talk is to review the key arguments and results of the paper [1]. The talk was given at the RTN workshop "The quantum structure of spacetime and the geometric nature of fundamental interactions" in Berlin, October 4-10, 2000.

Recently, new consistent supersymmetric theories have been discovered as limits of branes in the presence of near-critical electric fields. In $[5,6]$ the $p+1$ dimensional non-commutative open string $\left(\mathrm{NCOS}_{p+1}\right)$ theory was discovered as a decoupling limit of $\mathrm{D} p$-branes with a near-critical electrical NSNS two-form potential. In $[7,8]$ OM theory was discovered as a decoupling limit of the M5-brane with a near-critical electric threeform potential, and finally in $[1,7]$ the $\mathrm{OD} p$-theories ${ }^{1}$ were discovered as decoupling limits of the NS5-brane with near-critical electrical RR $(p+1)$-form potentials.

The interesting property that all these non-gravitational theories share is that they all have phases were the effective dynamics are governed by massive dynamical extended objects. Prior to the discovery of these new theories, the Little String Theory (LST) $[9,10]$ (see also $[11,12]$ ) was the only non-gravitational theory with this property. The hope is that these theories, being non-gravitational, eventually can be understod indendently of the critical string theories (this is already true for NCOS theories [6]). This might then eventually improve the understanding of string theory itself.

In this short review we briefly discuss NCOS theory and OM theory, and then move to the definition and properties of $(1,1)$ OBLST/OD1-theory, including the thermodynamics and the phase structure. We subsequently consider the $(2,0)$ OBLST/OD2-theory and finally we briefly look at other $\mathrm{OD} p$-theories.

\footnotetext{
${ }^{1}$ The OD1 and OD2-theories are called $(1,1)$ and $(2,0)$ OBLSTs in [1], as we discuss below.
} 


\section{Short review of NCOS and OM theory}

\subsection{NCOS theory}

NCOS theory was discovered in $[5,6]$. $\mathrm{NCOS}_{p+1}$ is obtained from the $\mathrm{F} 1-\mathrm{D} p$ bound state, i.e. a bound state of $\mathrm{F}$-strings dissolved in a $\mathrm{D} p$-brane. We take the $\mathrm{F}$-strings to be charged in the $x^{1}$ direction and the $\mathrm{D} p$-brane to be charged in the $x^{1}$ to $x^{p}$ directions.

The $\mathrm{NCOS}_{p+1}$ decoupling limit is ${ }^{2}$

$$
\begin{gathered}
l_{s} \rightarrow 0 \quad, \quad \varepsilon=\frac{l_{s}^{2}}{l_{\mathrm{os}}^{2}}, \quad g_{s}=\tilde{g} \varepsilon^{-1} \\
g_{\mu \nu}=\varepsilon^{-1} \eta_{\mu \nu} \quad, \quad \mu, \nu=0,1 \quad, \quad g_{i j}=\varepsilon \delta_{i j} \quad, \quad i, j=2, \ldots, 9
\end{gathered}
$$

where $l_{s}$ is the closed string length, $g_{s}$ is the closed string coupling and $g_{\mu \nu}$ is the closed string metric.

The limit (1)-(2) gives the NSNS two-form field $B_{\mu \nu}$ and RR $(p-1)$-form field $A_{\mu_{1} \cdots \mu_{p-1}}$

$$
B_{01}=\frac{1}{\varepsilon} \frac{1}{2 \pi l_{s}^{2}}-\frac{1}{4 \pi l_{\mathrm{os}}^{2}} \quad, \quad A_{2 \cdots p}=\frac{1}{(2 \pi)^{p-2} \tilde{g} l_{\mathrm{os}}^{p-1}}
$$

This can be obtained using the Seiberg-Witten relations between closed and open string moduli [13] together with the duality relation between $B_{01}$ and $A_{2 \ldots p}$.

If we have $N_{\mathrm{F} 1} \mathrm{~F}$-strings and $N_{\mathrm{D} p} \mathrm{D} p$-branes in the bound state we have the $\mathrm{RR}(p-1)$ form field from magnetic flux quantization $A_{2 \cdots p}=\frac{2 \pi}{N_{\mathrm{D} p}} \frac{N_{\mathrm{F} 1}}{V_{p-1}}$. where $V_{p-1}$ is the volume of the space with coordinates $x^{2}$ to $x^{p}$. If $V_{p-1}$ is infinite, we still have $\frac{N_{\mathrm{F} 1}}{V_{p-1}}$ finite. From this we get the NCOS coupling $\tilde{g}=\frac{V_{p-1}}{\left(2 \pi l_{\mathrm{los}}\right)^{p-1}} \frac{N_{\mathrm{D} p}}{N_{\mathrm{F} 1}}$.

We can see in a simple way that open F-string excitations can be light in the background field $B_{01}$, since the effective tension is $T_{\text {eff }}=\frac{1}{\varepsilon} \frac{1}{2 \pi l_{s}^{2}}-B_{01}=\frac{1}{4 \pi l_{o s}^{2}}$. The $\operatorname{NCOS}_{p+1}$ is a non-gravitational theory of open strings with only one orientation, and no closed strings when the $x^{1}$ directions is non-compact. From the Seiberg-Witten relations between closed and open string moduli [13] it can be seen that we have space-time non-commutativity $\left[x^{0}, x^{1}\right]=i 2 \pi l_{\text {os }}^{2}$. The theory reduces to a $p+1$ dimensional super Yang-Mills theory $\left(\mathrm{SYM}_{p+1}\right)$ at low energies with Yang-Mills coupling $g_{\mathrm{YM}}^{2}=(2 \pi)^{p-2} \tilde{g} l_{\mathrm{os}}^{p-3}$. The thermodynamics of NCOS theories have been considered in $[3,14,15,16]$.

\subsection{OM theory}

OM theory was discovered in $[7,8]$. OM theory is obtained from the M2-M5 bound state which is a bound state of M2-branes dissolved in an M5-brane. We take the M2-branes to be charged in the $x^{1}$ and $x^{2}$ directions and the M5-brane to be charged in the $x^{1}$ to $x^{5}$ directions. The OM theory decoupling limit is then

$$
\begin{gathered}
l_{p} \rightarrow 0, \quad f=\frac{l_{p}^{6}}{l_{\mathrm{om}}^{6}} \\
g_{\mu \nu}=f^{-1} \eta_{\mu \nu}, \quad \mu, \nu=0,1,2, \quad g_{i j}=f \delta_{i j}, \quad i, j=3, \ldots, 10
\end{gathered}
$$

\footnotetext{
${ }^{2}$ We use the $\operatorname{NCOS}_{p+1}$ limit used in [6] rather than the one used in [7].
} 
Using magnetic flux quantization and the self-duality of the three-form field strength on the M5-brane we get the three form potential

$$
C_{012}=\frac{1}{f} \frac{1}{(2 \pi)^{2} l_{s}^{3}}-\frac{1}{2(2 \pi)^{2} l_{\mathrm{om}}^{3}} \quad, \quad C_{345}=\frac{1}{(2 \pi)^{2} l_{\mathrm{om}}^{3}}
$$

along with $C_{345}=\frac{2 \pi}{N_{\mathrm{M} 5}} \frac{N_{\mathrm{M} 2}}{V_{3}}$ and $\frac{N_{\mathrm{M} 5}}{N_{\mathrm{M} 2}} \frac{V_{3}}{\left(2 \pi l_{\mathrm{om}}\right)^{3}}=1$ where $V_{3}$ is the volume of the space given by the $x^{3}$ to $x^{5}$ directions and where $N_{\mathrm{M} 2}$ and $N_{\mathrm{M} 5}$ are the number of M2 and M5-branes, respectively.

Open M2-branes are light in the background of the $C_{012}$ field when suitably oriented, since the effective membrane tension is $T_{\text {eff }}=\frac{1}{f} \frac{1}{(2 \pi)^{2} l_{p}^{3}}-C_{012}=\frac{1}{2(2 \pi)^{2} l_{\mathrm{om}}^{3}}$. OM theory is thus a non-gravitational theory of open membranes, that for $l_{\mathrm{om}} \rightarrow 0$ reduces to the $(2,0)$ SCFT.

When compactifying $\mathrm{OM}$ theory on an electric circle we get $\mathrm{NCOS}_{4+1}[7,8]$. An electric circle means a circle in one of the directions that the M2-branes are charged. Since this procedure requires identification of remaining part of the 11-dimensional metric with the 10-dimensional metric we require that $\varepsilon=f$. From this we get the relations $l_{\mathrm{om}}^{3}=R_{E} l_{\mathrm{os}}^{2}$ and $R_{E}=\tilde{g} l_{\text {os }}$ using the standard relations $f^{-1 / 2} R_{E}=g_{s} l_{s}$ and $l_{p}^{3}=f^{-1 / 2} R_{E} l_{s}^{2}$ with $R_{E}$ being the radius of the electric circle. The relation $l_{\mathrm{om}}^{3}=R_{E} l_{\mathrm{os}}^{2}$ means that the open string in NCOS theory comes from the open membrane in OM theory wrapped on the electric circle.

\section{$3 \mathrm{NCOS}_{5+1}$ theory and $(1,1)$ OBLST/OD1-theory}

\subsection{Why new theories?}

Some of the most important reasons that we need to consider the possibility of new theories, are

- As we shall see shortly, there are evidence for dynamical closed strings in the theory defined by the $\mathrm{NCOS}_{5+1}$ decoupling limit. These closed strings corresponds to the 'little' strings of ordinary LST, and they have a different tension than the open strings. This means we need to have a new theory that can have both an open string phase and a closed string phase.

- As has been considered in $[7,8,17]$, the $\operatorname{NCOS}_{p+1}$ theories for $p \leq 4$ can be obtained as the dimensional reduction of OM theory, just as $\mathrm{SYM}_{p+1}$ theories for $p \leq 4$ can be obtained as the dimensional reduction of $(2,0)$ SCFT. But the $\mathrm{SYM}_{5+1}$ and $(2,0)$ SCFT are connected through the $(2,0)$ and $(1,1)$ LSTs which reduce at low energies to $(2,0)$ SCFT and $\mathrm{SYM}_{5+1}$, respectively, and which are connected by T-duality. The question is if there are a similar type of connection between $\mathrm{NCOS}_{5+1}$ and OM theory through two new Little String Theories having also open branes in their spectra.

- If we consider thermal $\mathrm{NCOS}_{5+1}$ theory we can also ask what the high energy behavior of this can be. As was seen in $[3,14,16]$ the temperature can exceed the Hagedorn temperature of the NCOS theory. Therefore, the high energy behavior of $\mathrm{NCOS}_{5+1}$ theory cannot be described by weakly coupled NCOS theory.

All this points to the existence of two new theories generalizing the $(1,1)$ and $(2,0)$

LST, with 'little' strings. These new theories should be T-dual and should reduce to $\mathrm{NCOS}_{5+1}$ and OM theory when the 'little' strings are heavy. 


\subsection{S-duality on the $\mathrm{NCOS}_{5+1}$ limit}

Consider the $\mathrm{NCOS}_{5+1}$ limit of the F1-D5 bound state as in Section 2.1

$$
\begin{gathered}
\bar{l}_{s} \rightarrow 0 \quad, \quad \varepsilon=\frac{\bar{l}_{s}^{2}}{l_{\mathrm{os}}^{2}}, \quad \bar{g}_{s}=\tilde{g} \varepsilon^{-1} \\
g_{\mu \nu}=\varepsilon^{-1} \eta_{\mu \nu}, \mu, \nu=0,1 \quad, \quad g_{i j}=\varepsilon \delta_{i j}, \quad i, j=2, \ldots, 9 \\
B_{01}=\frac{1}{\varepsilon} \frac{1}{2 \pi \bar{l}_{s}^{2}}-\frac{1}{4 \pi l_{\mathrm{os}}^{2}}, \quad A_{2345}=\frac{1}{(2 \pi)^{3} \tilde{g} l_{\mathrm{os}}^{4}}
\end{gathered}
$$

Doing an S-duality transformation, this corresponds to the D1-NS5 bound state in the limit

$$
\begin{gathered}
g_{s} \rightarrow 0 \quad, \quad l_{s}=\text { fixed }, \quad \varepsilon=\frac{g_{s} l_{s}^{2}}{l_{\mathrm{os}}^{2}}=g_{s} \tilde{g} \\
g_{\mu \nu}=\varepsilon^{-1} \eta_{\mu \nu}, \quad \mu, \nu=0,1, \quad g_{i j}=\varepsilon \delta_{i j}, \quad i, j=2, \ldots, 9 \\
A_{01}=\frac{1}{\varepsilon} \frac{1}{2 \pi l_{s}^{2}}-\frac{1}{4 \pi l_{\mathrm{os}}^{2}}, \quad A_{2345}=\frac{1}{(2 \pi)^{3} \tilde{g} l_{\mathrm{os}}^{4}}
\end{gathered}
$$

where $l_{s}^{2}=\bar{g}_{s} \bar{l}_{s}^{2}$ and $g_{s}=1 / \bar{g}_{s}$. This limit of the D1-NS5 bound state gives an equivalent description of the theory since the limits are S-dual.

\section{3 'Little' closed strings}

As have already been described, the $\mathrm{NCOS}_{5+1}$ theory has open strings of tension $\frac{1}{4 \pi l_{\mathrm{ss}}^{2}}$ $[5,6]$. We are now ready to list the evidence for closed strings in the theory defined by the two S-dual decoupling limits (7)-(8) and (10)-(11):

- $\mathrm{NCOS}_{5+1}$ reduces to $\mathrm{SYM}_{5+1}$ for low energies, and $\mathrm{SYM}_{5+1}$ has closed string solitons of tension $\frac{(2 \pi)^{2}}{g_{\mathrm{YM}}^{2}}=\frac{1}{2 \pi l_{s}^{2}}$.

- In the limit $l_{\mathrm{os}} \rightarrow 0$ and $l_{s}=$ fixed the theory on D1-NS5 reduces to the usual LST on the NS5-brane with dynamical 'little' strings.

- As we review in Section 3.4, the thermodynamics calculated via the supergravity dual predicts Hagedorn behavior with a Hagedorn temperature given by the 'little' closed string scale. This means that the 'little' closed strings are dynamical.

We conclude that the theory on the F1-D5/D1-NS5 bound state contains 'little' closed strings of tension $\frac{1}{2 \pi l_{s}^{2}}$. We therefore have a new theory which encompass both $\operatorname{NCOS}_{5+1}$ and $(1,1)$ LST $[1,7]$. This theory is called $(1,1)$ open brane Little String Theory (OBLST) in [1] since it is almost the same as $(1,1)$ LST, the only difference is that the d0-particle in $(1,1)$ LST is replaced by the open string of $\mathrm{NCOS}_{5+1}$. In [7] it is called open D1brane (OD1) theory since in the D1-NS5 decoupling limit, the open brane origins from a D-string. More precisely, the effective tension of an open D-string stretching along the electrical field is $T_{\mathrm{eff}}=\frac{1}{\varepsilon} \frac{1}{2 \pi g_{s} l_{s}^{2}}-A_{01}=\frac{1}{4 \pi l_{\mathrm{os}}^{2}}$. The connection between the two string lengths and the NCOS coupling is $l_{s}^{2}=\tilde{g} l_{\mathrm{os}}^{2}$. 


\subsection{Supergravity dual, thermodynamics and phase diagrams}

The supergravity dual of $(1,1)$ OBLST/OD1-theory is found using the limit (7) on the F1-D5 bound state and the limit (10) on the D1-NS5, were the rescaling of the metric is replaced by an equivalent scaling of the coordinates. This is done in [1]. As shown in $[3,1,4]$ the thermodynamics computed from the F1-D5/D1-NS5 supergravity dual of $(1,1)$ OBLST/OD1-theory is

$$
T=T_{\mathrm{LST}} \quad, \quad F=0 \quad, \quad S=\frac{1}{T_{\mathrm{LST}}} E, \quad E=\frac{V_{5}}{(2 \pi)^{5}} \frac{\tilde{r}_{0}^{2}}{l_{\mathrm{os}}^{4} l_{s}^{4}}
$$

with

$$
T_{\mathrm{LST}}=\frac{1}{2 \pi l_{s} \sqrt{N}}
$$

where $T$ is the temperature, $F$ is the free energy, $E$ is the energy and $S$ is the entropy. Moreover, $V_{5}$ is the five-volume and $\tilde{r}_{0}=\frac{r_{0}}{\sqrt{\varepsilon}}$ where $r_{0}$ is the horizon radius of the nonextremal supergravity solution. This thermodynamics exhibits Hagedorn behavior with Hagedorn temperature $T_{\mathrm{LST}}$ as noticed in $[2,1,4]$ for this case and in $[18,19,2,20]$ for the ordinary LSTs.

Clearly, when the supergravity dual is a valid description of $(1,1)$ OBLST/OD1-theory we have $T \sim T_{\mathrm{LST}}$. Moreover, the 'little' closed strings dominates when the supergravity dual is valid since the Hagedorn temperature $T_{\mathrm{LST}}$ is given by the 'little' string scale [1].

For the ordinary LSTs it was found in $[2,20]$ that the entropy as function of temperature has the critical behavior

$$
S(T) \propto\left(T_{\mathrm{LST}}-T\right)^{-1}
$$

for $\left(T_{\mathrm{LST}}-T\right) / T_{\mathrm{LST}} \ll 1 / N^{3}$. This was found by adding one-loop string corrections to the temperature. In $[2,3,1,4]$ the critical behavior for OD1-theory was instead found to be

$$
S(T) \propto\left(T_{\mathrm{LST}}-T\right)^{-2 / 3}
$$

for $\left(T_{\mathrm{LST}}-T\right) / T_{\mathrm{LST}} \ll 1 / N^{3}$. This was found by doing tree-level string corrections to the temperature.

We see that the $(1,1)$ LST and the $(1,1)$ OBLST/OD1-theory have different critical behavior. This means that even when the 'little' strings are the dominant degrees of freedom the two theories are different. This we interpret to origin from having a different geometry which in the $(1,1)$ OBLST/OD1-theory should be a space-time non-commutative geometry with $\left[x^{0}, x^{1}\right]=i 2 \pi l_{\text {os }}^{2}$.

From the above we see that we have four phases of $(1,1)$ OBLST/OD1-theory:

- $\mathrm{SYM}_{5+1}$

- $\mathrm{NCOS}_{5+1}$

- $(1,1)$ LST

- $\mathrm{NC}(1,1) \mathrm{LST}$

where 'NC $(1,1)$ LST' means the phase of $(1,1)$ OBLST/OD1-theory where the 'little' strings dominates but the geometry supposedly is space-time non-commutative.

We now consider the phase diagrams of $(1,1)$ OBLST/OD1-theory. In the phase diagrams, we have defined the energy variable $u$ as $u=\frac{1}{l_{\mathrm{os}}^{2}} \frac{r}{\sqrt{\varepsilon}}$ where $r$ is the radial coordinate of the supergravity solution. 
The first case is $1 / N \ll \tilde{g} \ll 1$, depicted in figure 1 . In this case the NCOS theory is strongly coupled $\tilde{g} N \gg 1$ and the 'little' strings are light in the sense that $\frac{1}{N} \frac{1}{2 \pi l_{s}^{2}} \ll \frac{1}{2 \pi l_{o s}^{2}}$. The scale $\frac{1}{N} \frac{1}{2 \pi l_{s}^{2}}$ is not directly the 'little' string scale but it is the scale connected with the Hagedorn temperature $T_{\mathrm{LST}}$ and is therefore a scale that enters in the dynamics of 'little' strings (see $[2,1]$ for further discussion). We see that there are not any NCOS phase, which is sensible since it is strongly coupled and the NCOS scale is relatively heavy.

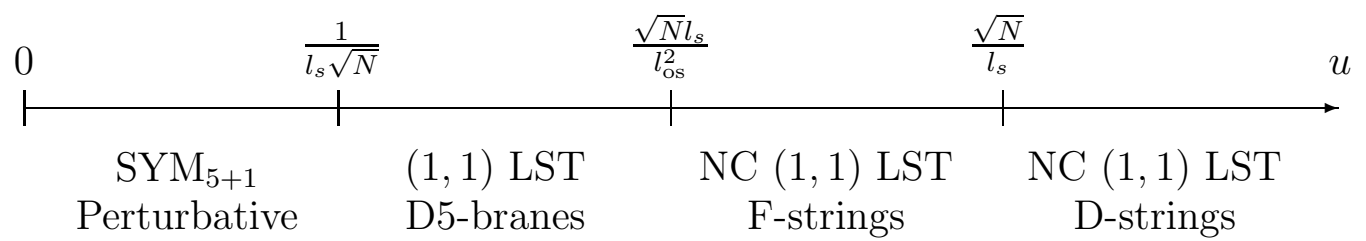

Figure 1: Phase diagram for $(1,1)$ OBLST/OD1-theory with $1 / N \ll \tilde{g} \ll 1$.

The second case is $\tilde{g} \gg 1$, depicted in figure 2 . Here we clearly have strong NCOS coupling $\tilde{g} \gg 1$ and the NCOS scale is large compared to the 'little' string scale $\frac{1}{2 \pi l_{s}^{2}} \ll$ $\frac{1}{2 \pi l_{\mathrm{os}}^{2}}$. Thus, we do not have any NCOS phase but instead a SYM phase, a LST phase and a non-commutative LST phase, which again is sensible since the 'little' strings are much lighter than the NCOS theory strings, and the NCOS theory is strongly coupled.

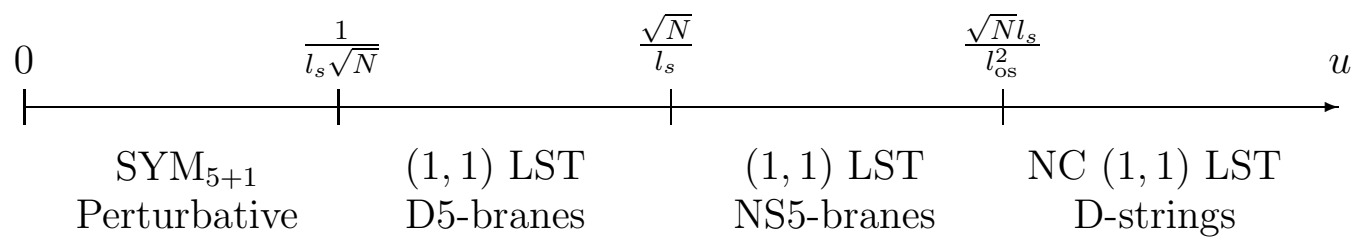

Figure 2: Phase diagram for $(1,1)$ OBLST/OD1-theory with $\tilde{g} \gg 1$.

The final case is $\tilde{g} \ll 1 / N$, depicted in figure 3 . Here we have a NCOS phase for low energies, which is sensible since the NCOS coupling is weak $\tilde{g} N \ll 1$ and since the NCOS scale is light $\frac{1}{2 \pi l_{o s}^{2}} \ll \frac{1}{N} \frac{1}{2 \pi l_{s}^{2}}$. But, we still enter a non-commutative LST phase at high energies. This can be explained by the fact that the Hagedorn temperature of NCOS theory is $T_{\mathrm{NCOS}} \sim \frac{1}{l_{\mathrm{os}}}$ so that $T_{\mathrm{NCOS}} \ll T_{\mathrm{LST}}$. This means that there must be an NCOS theory Hagedorn transition before $u$ reaches $\frac{1}{l_{\mathrm{os}}}$ since for $u \gg \frac{1}{l_{\mathrm{os}}}$ we have $T \sim T_{\mathrm{LST}}$. Thus, the non-commutative LST phase comes after the NCOS theory has been subject to a Hagedorn transition.

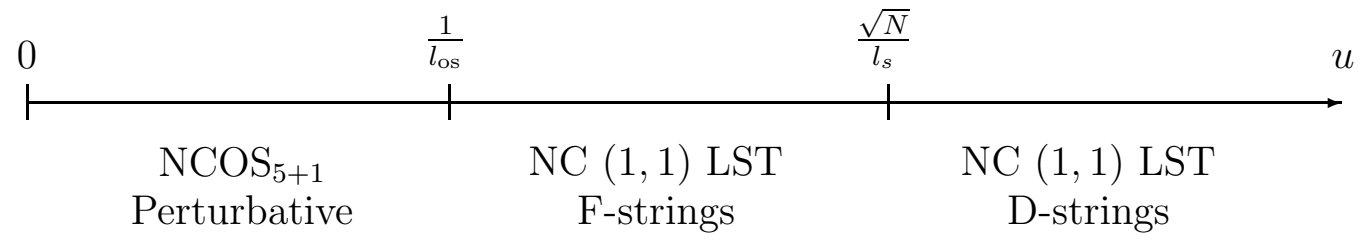

Figure 3: Phase diagram for $(1,1)$ OBLST/OD1-theory with $\tilde{g} \ll 1 / N$. 


\section{OM theory and $(2,0)$ OBLST/OD2-theory}

\subsection{Motivation and definition}

Consider the $(1,1)$ OBLST/OD1-theory. This is obtained from the D1-NS5 bound state. But T-dualizing this we get D2-NS5, which is nothing but M2-M5 with a compact transverse direction. Thus, defining a T-dual theory of the $(1,1)$ OBLST/OD1-theory in this way we can get a theory that can reduce to OM-theory for low energies. This provides a nice connection via dualities between $\mathrm{NCOS}_{5+1}$ and OM theory.

Thus, we define a new theory by the OM theory limit (4)-(5) on the M2-M5 boundstate with a transverse circle of radius $R_{T}$. When $R_{T}$ is small we can use a type IIA description in terms of a D2-NS5 bound state in the limit

$$
\begin{gathered}
\hat{g}_{s} \rightarrow 0 \quad, \quad l_{s}=\text { fixed }, \quad f=\frac{\hat{g}_{s}^{2} l_{s}^{6}}{l_{\mathrm{om}}^{6}} \\
g_{\mu \nu}=f^{-1} \eta_{\mu \nu}, \quad \mu, \nu=0,1,2, \quad g_{i j}=f \delta_{i j}, \quad i, j=3, \ldots, 9 \\
A_{012}=\frac{1}{f} \frac{1}{(2 \pi)^{2} l_{s}^{3}}-\frac{1}{2(2 \pi)^{2} l_{\mathrm{om}}^{3}} \quad, \quad A_{345}=\frac{1}{(2 \pi)^{2} l_{\mathrm{om}}^{3}}
\end{gathered}
$$

This new theory on the D2-NS5 bound state is called $(2,0)$ OBLST in [1] since it is almost like $(2,0)$ LST, but with an open membrane instead of the d1-brane in $(2,0)$ LST. In [7] it is called open D2-brane (OD2) theory since the open membrane origins from an open D2-brane. The open membrane has the tension $T_{\text {eff }}=\frac{1}{f} \frac{1}{(2 \pi)^{2} g_{s} l_{s}^{3}}-A_{012}=\frac{1}{2(2 \pi)^{2} l_{\mathrm{om}}^{3}}$. The $(2,0)$ OBLST/OD2-theory has 'little' strings of tension $\frac{1}{2 \pi l_{s}^{2}}$.

\subsection{T-duality to $(1,1)$ OBLST/OD1-theory}

We can now consider doing a T-duality transformation on $(2,0)$ OBLST/OD2-theory in an electric direction. Choose the direction of $x^{2}$ to be on a circle of radius $R_{E}$. Using the standard T-duality transformation

$$
g_{s}=\hat{g}_{s} \frac{l_{s}}{f^{-1 / 2} R_{E}} \quad, \quad R_{E} R_{E}^{\prime}=l_{s}^{2} \quad, \quad g_{22} g_{22}^{\prime}=1
$$

we see from the limits (10)-(11) and (17)-(18) that the T-duality works if and only if $f=\varepsilon$ and $l_{\mathrm{om}}^{3}=R_{E} l_{\mathrm{os}}^{2}$. From this we get

$$
2 \pi R_{E} \frac{1}{2(2 \pi)^{2} l_{\mathrm{om}}^{3}}=\frac{1}{4 \pi l_{\mathrm{os}}^{2}}
$$

so that the tension of the open string is equal to the tension of the wrapped open membrane. Thus, the open membrane and the open string are T-dual to each other. This provides a new connection between $\mathrm{NCOS}_{5+1}$ and OM theory in the sense that their fundamental objects are T-dual.

\subsection{Phase diagrams}

The thermodynamics of $(2,0)$ OBLST/OD2-theory computed from the supergravity dual plus string correction terms is the same as the one reviewed for $(1,1)$ OBLST/OD1-theory in 3.4 .

We have again four different phases 
- $(2,0)$ SCFT

- OM theory

- $(2,0) \mathrm{LST}$

- NA $(2,0)$ LST

'NA $(2,0)$ LST' refers to the phase of $(2,0)$ OBLST/OD2-theory where the 'little' strings dominate but the geometry supposedly is non-associative. We get two phase diagrams, depicted in figure 4 and 5 , where $\tilde{r}=r / \sqrt{f}$ with $r$ being the radial coordinate in the M2-M5/D2-NS5 supergravity solution [1] (see also [21]).

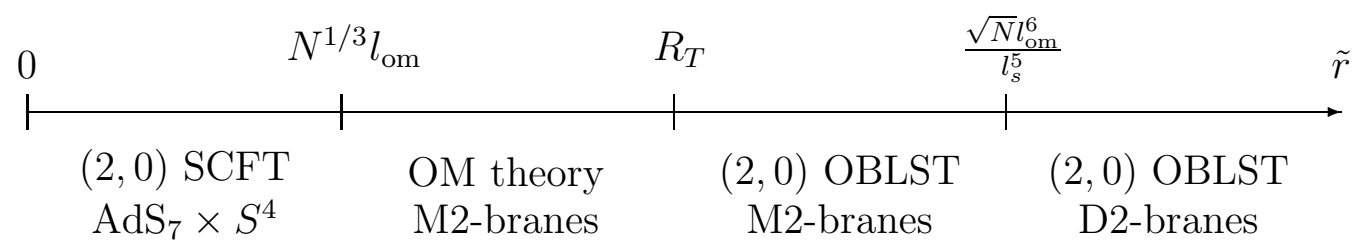

Figure 4: Phase diagram for $(2,0)$ OBLST/OD2-theory.

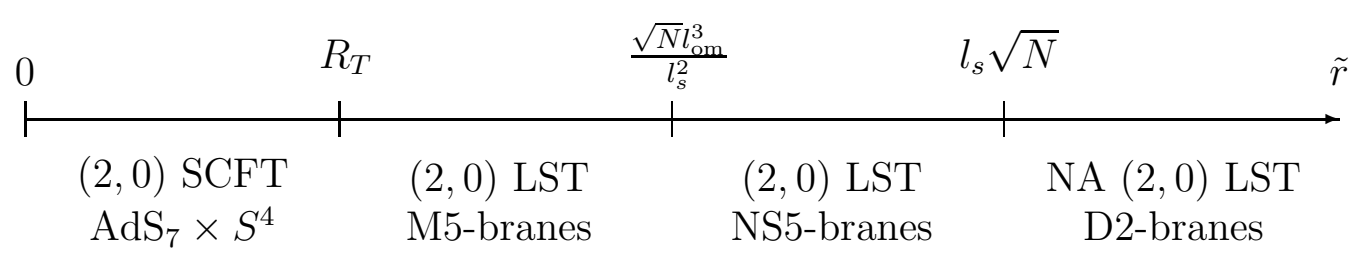

Figure 5: Phase diagram for $(2,0)$ OBLST/OD2-theory.

\section{Other $\mathrm{OD} p$-theories}

The OD1 and OD2-theories were discovered in [1, 7], but in [7] there were in addition discovered other $\mathrm{OD} p$-theories. The $\mathrm{OD} p$-theories, $p=0,1, \ldots, 5$, are defined via the limit[7]

$$
\begin{gathered}
l_{s} \rightarrow 0 \quad, \quad \varepsilon=\frac{l_{s}^{4}}{l_{\mathrm{eff}}^{4}}, \quad g_{s}=\tilde{g} \varepsilon^{\frac{3-p}{4}} \\
g_{\mu \nu}=\eta_{\mu \nu}, \mu, \nu=0,1, \ldots, p, \quad g_{i j}=\varepsilon \delta_{i j}, \quad, \quad i, j=p+1, \ldots, 9 \\
A_{01 \cdots p}=\frac{1}{(2 \pi)^{p} g_{s} l_{s}^{p+1}}-\frac{1}{2(2 \pi)^{p} \tilde{g} l_{\mathrm{eff}}^{p+1}} \quad, \quad A_{p+1 \ldots 5}=\frac{1}{(2 \pi)^{4-p} \tilde{g} l_{\mathrm{eff}}^{5-p}}
\end{gathered}
$$

The OD $p$-theories have 'little' strings of tension $\frac{1}{2 \pi l_{\text {eff }}^{2}}$ and open $p$-branes of tension $T_{\text {eff }}=\frac{1}{(2 \pi)^{p} g_{s} l_{s}^{p+1}}-A_{01 \cdots p}=\frac{1}{2(2 \pi)^{p} \tilde{g} l_{\text {eff }}^{p+1}}$. The supergravity duals of OD $p$-theories are given in $[22,4]$ for all $p=0,1, \ldots, 5$. Their thermodynamics computed from supergravity plus string corrections have been studied in [4] for all $p=0,1, \ldots, 5$.

\section{Acknowledgments}

We thank the organizers for a nice and fruitful conference. We thank Niels Obers for collaboration and many useful discussions. We also thank Jan de Boer and Robbert Dijkgraaf for many interesting discussions and comments. 


\section{References}

[1] T. Harmark, "Open branes in space-time non-commutative little string theory," Nucl. Phys. B593 (2001) 76-98, hep-th/0007147.

[2] T. Harmark and N. A. Obers, "Hagedorn behaviour of little string theory from string corrections to NS5-branes," Phys. Lett. B485 (2000) 285, hep-th/0005021.

[3] T. Harmark, "Supergravity and space-time non-commutative open string theory," JHEP 07 (2000) 043, hep-th/0006023.

[4] T. Harmark and N. A. Obers, "Hagedorn behavior of little string theories," hep-th/0010169.

[5] N. Seiberg, L. Susskind, and N. Toumbas, "Strings in background electric field, space / time noncommutativity and a new noncritical string theory," JHEP 06 (2000) 021, hep-th/0005040.

[6] R. Gopakumar, J. Maldacena, S. Minwalla, and A. Strominger, "S-duality and noncommutative gauge theory," JHEP 06 (2000) 036, hep-th/0005048.

[7] R. Gopakumar, S. Minwalla, N. Seiberg, and A. Strominger, "OM theory in diverse dimensions," JHEP 08 (2000) 008, hep-th/0006062.

[8] E. Bergshoeff, D. S. Berman, J. P. van der Schaar, and P. Sundell, "Critical fields on the M5-brane and noncommutative open strings," hep-th/0006112.

[9] M. Berkooz, M. Rozali, and N. Seiberg, "Matrix description of M theory on $T^{4}$ and T5," Phys. Lett. B408 (1997) 105-110, hep-th/9704089.

[10] N. Seiberg, "New theories in six-dimensions and matrix description of $\mathrm{M}$ theory on $T^{5}$ and $T^{5} / Z_{2}, "$ Phys. Lett. B408 (1997) 98-104, hep-th/9705221.

[11] R. Dijkgraaf, E. Verlinde, and H. Verlinde, "BPS spectrum of the five-brane and black hole entropy," Nucl. Phys. B486 (1997) 77-88, hep-th/9603126.

[12] R. Dijkgraaf, E. Verlinde, and H. Verlinde, "BPS quantization of the five-brane," Nucl. Phys. B486 (1997) 89-113, hep-th/9604055.

[13] N. Seiberg and E. Witten, "String theory and noncommutative geometry," JHEP 09 (1999) 032, hep-th/9908142.

[14] I. R. Klebanov and J. Maldacena, " $1+1$ dimensional NCOS and its U(N) gauge theory dual," hep-th/0006085.

[15] V. Sahakian, "The phases of 2-D NCOS," JHEP 09 (2000) 025, hep-th/0008073.

[16] S. S. Gubser, S. Gukov, I. R. Klebanov, M. Rangamani, and E. Witten, "The Hagedorn transition in non-commutative open string theory," hep-th/0009140.

[17] T. Kawano and S. Terashima, "S-duality from OM-theory," hep-th/0006225.

[18] J. M. Maldacena, "Statistical entropy of near extremal five-branes," Nucl. Phys. B477 (1996) 168-174, hep-th/9605016.

[19] J. M. Maldacena and A. Strominger, "Semiclassical decay of near-extremal fivebranes," JHEP 12 (1997) 008, hep-th/9710014. 
[20] M. Berkooz and M. Rozali, "Near Hagedorn dynamics of NS fivebranes, or a new universality class of coiled strings," JHEP 05 (2000) 040, hep-th/0005047.

[21] M. Alishahiha, "On type II NS5-branes in the presence of an RR field," hep-th/0002198.

[22] M. Alishahiha, Y. Oz, and J. G. Russo, "Supergravity and light-like noncommutativity," hep-th/0007215. 